\title{
Thermoresponsive copolymer-grafted SBA-15 porous silica particles for temperature-triggered topical delivery systems
}

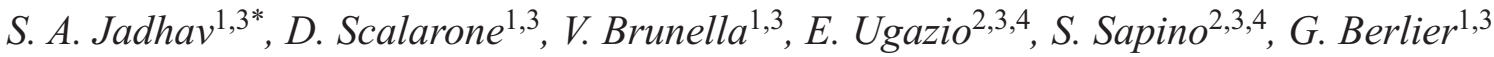 \\ ${ }^{1}$ Department of Chemistry, University of Torino, 10125 Torino, Italy \\ ${ }^{2}$ Department of Pharmaceutical Science and Technology, University of Torino, 10125 Torino, Italy \\ ${ }^{3}$ NIS Research Centre, University of Torino, 10125 Torino, Italy \\ ${ }^{4} \mathrm{G}$. Scansetti, Interdepartmental Centre, University of Torino, 10125 Torino, Italy
}

Received 9 July 2016; accepted in revised form 23 September 2016

\begin{abstract}
A series of poly( $N$-isopropylacrylamide- $c o$-acrylamide) thermoresponsive random copolymers with different molecular weights and composition were synthesized and characterized by attenuated total reflectance Fourier-transform infrared (ATR-FTIR), differential scanning calorimetry (DSC), size exclusion chromatography (SEC) and proton nuclear magnetic resonance (NMR) spectroscopy. The lower critical solution temperatures (LCST) of the copolymers were tuned by changing the mole ratios of monomers. Copolymer with highest molecular weight and LCST $\left(41.2^{\circ} \mathrm{C}\right)$ was grafted on SBA-15 type mesoporous silica particles by a two-step polymer grafting procedure. Bare SBA-15 and the thermoresponsive copolymergrafted (hybrid) SBA-15 particles were fully characterized by scanning electron microscope (SEM), ATR-FTIR, thermogravimetric analysis (TGA) and Brunauer-Emmett-Teller (BET) analyses. The hybrid particles were tested for their efficiency as temperature-sensitive systems for dermal delivery of the antioxidant rutin (quercetin-3-O-rutinoside). Improved control over rutin release by hybrid particles was obtained which makes them attractive hybrid materials for drug delivery.
\end{abstract}

Keywords: smart polymers, lower critical solution temperature, nanomaterial, SBA-15 particles, temperature-triggered delivery to skin

\section{Introduction}

Thermoresponsive polymers, especially poly $(N$-isopropylacrylamide) (PNIPAM) and its copolymers are investigated with great interest for their applications in biomedical fields [1-5]. These polymers in aqueous solution show a typical coil-to-globule transition above its lower critical solution temperature (LCST), that is upon heating the water soluble extended chains of the polymer collapse to form compact insoluble globules [6, 7]. This property of PNIPAM and its copolymers is used for designing of hybrid thermoresponsive drug delivery systems (DDS). In order to adjust the hydrophobicity of PNIPAM and thereby its LCST various random and block copolymers of $\mathrm{N}$-isopropylacrylamide (NIPAM) with different composition and microstructure have been synthesized $[8,9]$. Copolymerization of NIPAM with different monomers thus allows tuning of the properties especially LCST of PNIPAM according to the requirements of the applications under investigation. There is increased interest in hybrid thermoresponsive DDS as several studies reported their synthesis and testing for the delivery of various drugs in different environments and physiological conditions, including dermal drug delivery and also their use in dermocosmetic applications [10]. For the preparation of hybrid thermosensitive DDS, the thermoresponsive polymer needs to be anchored on a solid support. For instance, grafting of thermoresponsive polymers on porous particles leads to the formation of hybrid

*Corresponding author, e-mail: sushil.unige@gmail.com (C) BME-PT 
thermosensitive porous materials useful in temperature-triggered drug delivery $[11,12]$. The porous particles in such DDS act as reservoirs for various molecules and the thermoresponsive polymer, through its typical coil-to-globule transition at its LCST, applies a pore opening and closing mechanism to the porous particles $[13,14]$. This mechanism helps in the temperature-triggered release of the loaded molecules by the hybrid DDS.

The main difficulty in the use of PNIPAM homopolymer in the preparation of topical delivery systems is its LCST which is around $31-33^{\circ} \mathrm{C}$. This transition temperature is very close to the human skin, hence they are not efficient. As the LCST of PNIPAM is low, an uncontrolled release of the loaded drug is observed due to immediate transition of PNIPAM when it comes in contact with the skin. To address this important issue, in the present study first we report synthesis of a series of random copolymers of NIPAM with acrylamide (AM) to tune the LCST to higher values. Later grafting of the copolymer on SBA-15 type mesoporous silica particles was carried out. The higher values of LCST $\left(41-42^{\circ} \mathrm{C}\right)$ obtained for the copolymers can help to trigger the thermoresponsive transition of the copolymer with an external mild heating device. The hybrid thermoresponsive copolymergrafted porous particles were tested for their efficiency in the temperature-triggered delivery of rutin. The flavonoid rutin is believed to exhibit significant pharmacological activities, including anti-oxidation, antiinflammation, anti-diabetic, anti-adipogenic, and in hormone therapy $[15,16]$. The in vitro tests showed improved control over the release of the loaded rutin by the hybrid particles with respect to the bare porous silica particles. Thus the present work is an important step towards transformation of conventional porous materials used for drug delivery into hybrid thermoresponsive DDS with improved properties. It is believed that the same synthetic strategy presented in the work can be applied to various porous micro and nanoparticles to transform them into thermoresponsive delivery systems, which further increases the general scope of the study.

\section{Experimental \\ 2.1. Materials}

$\mathrm{N}$-isopropylacrylamide (NIPAM), acrylamide (AM), 3-(methacryloxypropyl) trimethoxysilane (MPS), azobisisobutyronitrile (AIBN) and rutin were purchased from Sigma-Aldrich, Italy. Porous silica particles of
SBA-15 type used in this study were purchased from Zecasin S.A., Romania. The chemical analysis of particles provided by the producer was $\mathrm{SiO}_{2}$ (wt $\%$ 99.91 ) and $\mathrm{Na}_{2} \mathrm{O}$ (wt\% 0.005). All the solvents mentioned in the experimental procedure were purchased from Sigma-Aldrich, Italy and were of high purity and used as received.

\subsection{Instruments and methods}

Fourier-transform infrared (FTIR) spectra were recorded on a Perkin Elmer Spectrum 100 in the attenuated total reflectance (ATR) mode with a diamond crystal, using 32 scans per spectrum, a resolution of $4 \mathrm{~cm}^{-1}$ and a spectral range of $4000-600 \mathrm{~cm}^{-1}$. Proton nuclear magnetic resonance (NMR) spectra were recorded on a Bruker Avance NMR $(200 \mathrm{MHz})$ spectrometer in deuterated dimethyl sulfoxide (DMSO-D 6 ) with tetramethylsilane (TMS) as reference. Thermogravimetric analyses (TGA) were carried out on a TA Q500 model from TA Instruments by heating samples contained in alumina pans at a rate of $10^{\circ} \mathrm{C} \cdot \mathrm{min}^{-1}$ from 25 to $600^{\circ} \mathrm{C}$ in a nitrogen flow and from 600 to $800^{\circ} \mathrm{C}$ in air. Change of the gas at $600^{\circ} \mathrm{C}$ was used to remove completely the carbonaceous residues from pyrolysis reactions and measure the exact amount of organic component.

The cloud points of the polymers were measured using a digital thermometer by heating the aqueous solution of the polymers. A differential scanning calorimeter (DSC Q200, TA Inc.) was used to collect DSC thermograms of the copolymers in water solution. $50 \mathrm{mg}$ of polymer were dissolved in $0.5 \mathrm{~mL}$ of water, a couple of drops were poured in an aluminum pan and DSC measurements were performed under nitrogen atmosphere with heating rate of $10^{\circ} \mathrm{C} / \mathrm{min}$, from 20 up to $60^{\circ} \mathrm{C}$.

Size exclusion chromatography (SEC) was performed with a Viscotek modular instrument equipped with a VE 1122 pump, a VE 7510 degasser, manual injection valve, VE 3580 refractive index detector, column oven and two PLgel $10 \mu \mathrm{m}$ MIXED-B columns (Polymer Laboratories). $N, N$-Dimethylformamide $\left(1.0 \mathrm{~mL} \cdot \mathrm{min}^{-1}\right)$ was used as eluent and analyses were performed setting the column oven at $70^{\circ} \mathrm{C}$. DMF solutions of the samples $(3 \mathrm{mg} / \mathrm{mL})$ were filtered through $0.45 \mu \mathrm{m}$ PTFE membrane filters. Calibration was obtained with PMMA molecular weight standards.

Scanning electron microscope (SEM) images were obtained with SEM Zeiss Evo-50 instrument equipped 
with a secondary electron detector. The samples were previously coated with gold by a sputter coater (Baltec SCD 050) for $60 \mathrm{~s}$ under vacuum at a current intensity of $60 \mathrm{~mA}$ after mounting the sample on an aluminum stub with double-sided conductive tape. Gas-volumetric analysis, specific surface area (SSA), pore volume and size were measured by $\mathrm{N}_{2}$ adsorption-desorption isotherms at $-196^{\circ} \mathrm{C}$ using an ASAP 2020 (Micromeritics) gas-volumetric analyzer. SSA was calculated using the Brunauer-Emmett-Teller (BET) method; average pore size and volume were calculated on the adsorption branch of the isotherms according to the Barrett-Joyner-Halenda (BJH) method (Kruk-Jaroniec-Sayari equations). Prior to analyses, samples were outgassed at room temperature (RT) overnight.

\subsection{Synthesis}

\subsubsection{Synthesis of poly(NIPAM-co-AM) copolymers}

Solution of weighted quantities of monomers in $3 \mathrm{~mL}$ degassed anhydrous 1,4-dioxane was prepared in a two-neck round-bottom flask equipped with heating bath and nitrogen balloon. Solution of initiator (AIBN) prepared in $1 \mathrm{~mL}$ of degassed 1,4-dioxane was injected into the flask with monomer solution and the polymerization reaction was continued for $5 \mathrm{~h}$ at $70^{\circ} \mathrm{C}$ under nitrogen atmosphere. The obtained polymers were recovered by pouring the reaction mixture on ice cold ether. Then polymers were reprecipitated from cold ether. All the polymer samples were dried under vacuum at $30^{\circ} \mathrm{C}$ for $5-6 \mathrm{~h}$ before characterization. Table 1 enlists the prepared samples and Table 2 reports the corresponding characterization data.

\subsubsection{Synthesis of SBA-MPS grafted particles}

(Figure 4a first step)

Suspension of $500 \mathrm{mg}$ of SBA-15 particles in $18 \mathrm{~mL}$ toluene was prepared in a round-bottom flask, to it $50 \mu \mathrm{L}$ of MPS was injected. The suspension was then

Table 1. List of synthesized random copolymers

\begin{tabular}{|c|c|c|c|}
\hline Sample & $\begin{array}{c}\text { NIPAM fraction } \\
\text { [mol\%] }\end{array}$ & $\begin{array}{c}\text { AM fraction } \\
\text { [mol\%] }\end{array}$ & $\begin{array}{c}\text { NIPAM/AIBN } \\
\text { [mole ratio] }\end{array}$ \\
\hline Copolymer 1 & 94.0 & 6.0 & $10 / 1$ \\
\hline Copolymer 2 & 94.0 & 6.0 & $30 / 1$ \\
\hline Copolymer 3 & 94.0 & 6.0 & $50 / 1$ \\
\hline Copolymer 4 & 94.0 & 6.0 & $100 / 1$ \\
\hline Copolymer 5 & 88.2 & 11.8 & $10 / 1$ \\
\hline Copolymer 6 & 88.2 & 11.8 & $50 / 1$ \\
\hline Copolymer 7 & 88.2 & 11.8 & $100 / 1$ \\
\hline
\end{tabular}

sonicated for 15-20 min to allow the diffusion of MPS molecules inside the pores. The flask was then equipped with water condenser and nitrogen balloon and heated at reflux with magnetic stirring at $125^{\circ} \mathrm{C}$ for $16 \mathrm{~h}$. At the end of the reaction, the suspension was cooled to RT and centrifuged to separate the particles. Particles were washed once with fresh $5 \mathrm{~mL}$ toluene and twice with $5 \mathrm{~mL}$ ethanol. Each time the particles were separated from the suspension by centrifugation. The obtained final product was then dried in vacuum for several hours to remove solvent traces.

\subsubsection{Synthesis of poly(NIPAM-co-AM)/SBA hybrid particles (Figure 4a second step)}

Reaction conditions and mole ratio of monomers were the same used for copolymer 7 from Table 1. In a two-neck round-bottom flask, a solution of $112.5 \mathrm{mg}$ of NIPAM and $9.5 \mathrm{mg}$ of AM was prepared in $3 \mathrm{~mL}$ of anhydrous and degassed 1,4-dioxane. To it $250 \mathrm{mg}$ of SBA-MPS particles were added. The resulting suspension was sonicated for 1520 min. The solution of initiator AIBN (NIPAM/ AIBN, 100/1 mole ratio) in $1 \mathrm{~mL}$ of anhydrous 1,4dioxane was prepared in a separate vial. The roundbottom flask was then kept in heating bath at $70^{\circ} \mathrm{C}$ equipped with a water condenser, magnetic stirrer and nitrogen balloon. The solution of the initiator was injected into the flask. The polymerization reaction was continued for $5 \mathrm{~h}$ at $70^{\circ} \mathrm{C}$ under nitrogen. At the end of the reaction, the suspension in the flask was cooled and centrifuged to obtain polymer-grafted particles. Particles were washed twice with acetone to remove solvent traces. The polymer-grafted particles were then dried in vacuum for several hours before characterization.

\subsection{Loading and release tests}

Loading of model antioxidant drug rutin inside the pores of SBA-15 and poly(NIPAM-co-AM)/SBA particles was carried out by wet mixing method. In a typical procedure the particles were mixed with rutin in $1 / 1$ weight ratio in $1 \mathrm{~mL}$ of ethanol and mixed well. The obtained complex was dried in desiccator to remove the solvent. The complex was washed quickly with ethanol to remove physisorbed rutin. Quantitative loading of rutin was determined by extraction with DMSO, which is its best solvent. Loading conditions and results are reported in Table 3.

For release tests a weighted amount of hybrid particles loaded with rutin were dispersed in $25 \mathrm{~mL}$ of 
phosphate buffer (pH 6.5)/DMSO (75/25). These dispersions were placed in incubator at $20^{\circ} \mathrm{C}$ (below LCST) and $45^{\circ} \mathrm{C}$ (above LCST). At a predetermined time interval, $1.0 \mathrm{~mL}$ aliquots from the dispersions were withdrawn and replaced with fresh release media. After centrifugation at 16000 rotation per minute (r.p.m.) for $10 \mathrm{~min}$, the concentrations of the released molecules in the supernatant were determined via UV-visible spectrophotometry at $\lambda_{\max }=$ $260 \mathrm{~nm}$. The results of the release tests are reported as percentage of released rutin vs time.

\section{Results and discussion}

\subsection{Synthesis of poly(NIPAM-co-AM) copolymers}

Random copolymers of NIPAM and AM were synthesized by standard radical polymerization method using AIBN as the initiator $[17,18]$. The characterization data of the synthesized polymers are shown in Table 2. Molecular weights of the copolymers were in the range of 97300 to $172300 \mathrm{Da}$. As expected, molecular weights of the copolymers increased with increasing the monomer to initiator ratio. LCSTs of the copolymers were increased from 37 to $41^{\circ} \mathrm{C}$ with increase of AM mole fraction from 6.0 to $11.8 \%$. Representative ATR-FTIR spectra of copolymers 2 and 7 (one from each series of AM fraction) are shown in Figure 1. The characteristic peaks of NIPAM and AM units are evident. Peaks at 3445, 3441, 3319, and $3318 \mathrm{~cm}^{-1}$ are attributed to the asymmetric stretching of the $-\mathrm{NH}_{2}$ group from $\mathrm{AM}$ and $-\mathrm{N}-\mathrm{H}$ stretching from NIPAM units, respectively [19]. Peaks at 1650 and $1545 \mathrm{~cm}^{-1}$ in both spectra are due to the carbonyl $(\mathrm{C}=\mathrm{O})$ group stretching and $-\mathrm{N}-\mathrm{H}$ bending in secondary amines [19]. These data confirmed the

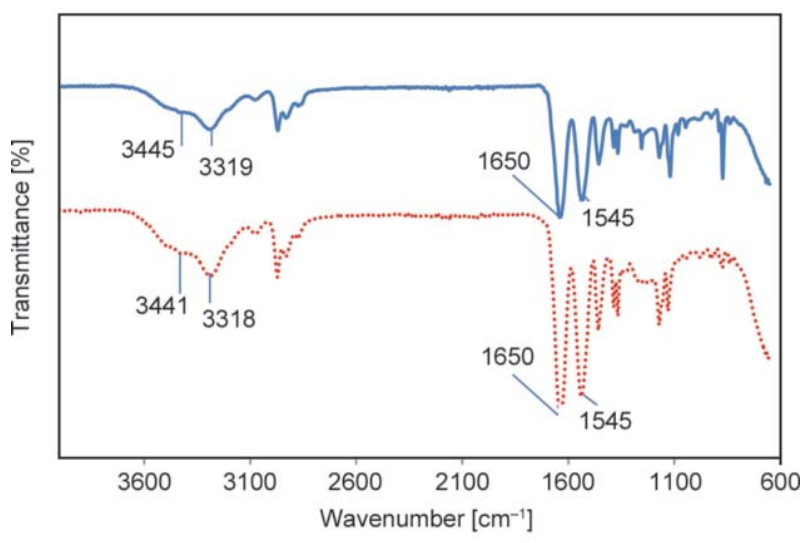

Figure 1. ATR-FTIR spectra of copolymers 2 (solid line) and 7 (dotted line)

formation of the copolymers and the presence of groups from both structural units.

From the characterization data of the copolymers it was observed that copolymer 7 shows desired properties with highest molecular weight and cloud point around $41^{\circ} \mathrm{C}$. High molecular weight of the copolymer 7 can guarantee good surface coverage and helps to apply the pore opening and closing mechanism to the pores on the particles. Furthermore, a cloud point of $41^{\circ} \mathrm{C}$, being significantly higher than the skin temperature, is suitable for cutaneous applications. Due to this, the composition and polymerization conditions used for the synthesis of copolymer 7 were chosen to prepare the copolymer-grafted SBA-15 particles. Copolymer 7 was subjected to additional structural and thermal characterization by NMR spectroscopy and DSC analysis, respectively. The NMR spectrum of copolymer 7 is shown in Figure 2. It shows characteristic signals for all the protons present in the copolymer and is well in agreement with the reported data [20,21]. The feed composition cal-

Table 2. Characterization data of copolymers

\begin{tabular}{|l|c|c|c|c|c|}
\hline \multicolumn{1}{|c|}{ Sample } & $\begin{array}{c}\text { AM fraction } \\
{[\mathbf{m o l} \% \mathbf{0}}\end{array}$ & $\begin{array}{c}\boldsymbol{M}_{\mathbf{w}} \\
{[\mathbf{D a}]}\end{array}$ & $\begin{array}{c}\boldsymbol{M}_{\mathbf{n}} \\
{[\mathbf{D a}]}\end{array}$ & $\boldsymbol{M}_{\mathbf{w}} / \boldsymbol{M}_{\mathbf{n}}$ & $\begin{array}{c}\text { Cloud point in } \\
\mathbf{w a t e r} \pm \mathbf{0 . 2}\end{array}$ \\
\hline Copolymer 1 & 107000 & 37200 & 2.87 & 37 \\
\hline Copolymer 2 & 6.0 & 108800 & 46300 & 2.35 & 37 \\
\hline Copolymer 3 & 6.0 & 112600 & 51000 & 2.21 & 37 \\
\hline Copolymer 4 & 6.0 & 164400 & 62000 & 2.65 & 37 \\
\hline Copolymer 5 & 6.0 & 97300 & 29500 & 3.30 & 41 \\
\hline Copolymer 6 & 11.8 & 162600 & 67200 & 2.42 & 41 \\
\hline Copolymer 7 & 11.8 & 172300 & 69000 & 2.50 & 41 \\
\hline
\end{tabular}

Table 3. Drug loading conditions and values

\begin{tabular}{|l|c|c|c|}
\hline \multicolumn{1}{|c|}{ Sample } & Silica:Drug weight ratio, loading conditions & Extraction conditions & Loading by UV-Vis. $\pm \mathbf{1 \%}$ \\
\hline SBA-Rutin & $1 / 1$, in ethanol, $20^{\circ} \mathrm{C}, 24 \mathrm{~h}$ & DMSO $, 45^{\circ} \mathrm{C}, 24 \mathrm{~h}$ & 35.3 \\
\hline SBA-copolymer-Rutin & $1 / 1$, in ethanol, $20^{\circ} \mathrm{C}, 24 \mathrm{~h}$ & DMSO, $45^{\circ} \mathrm{C}, 24 \mathrm{~h}$ & 27.9 \\
\hline
\end{tabular}




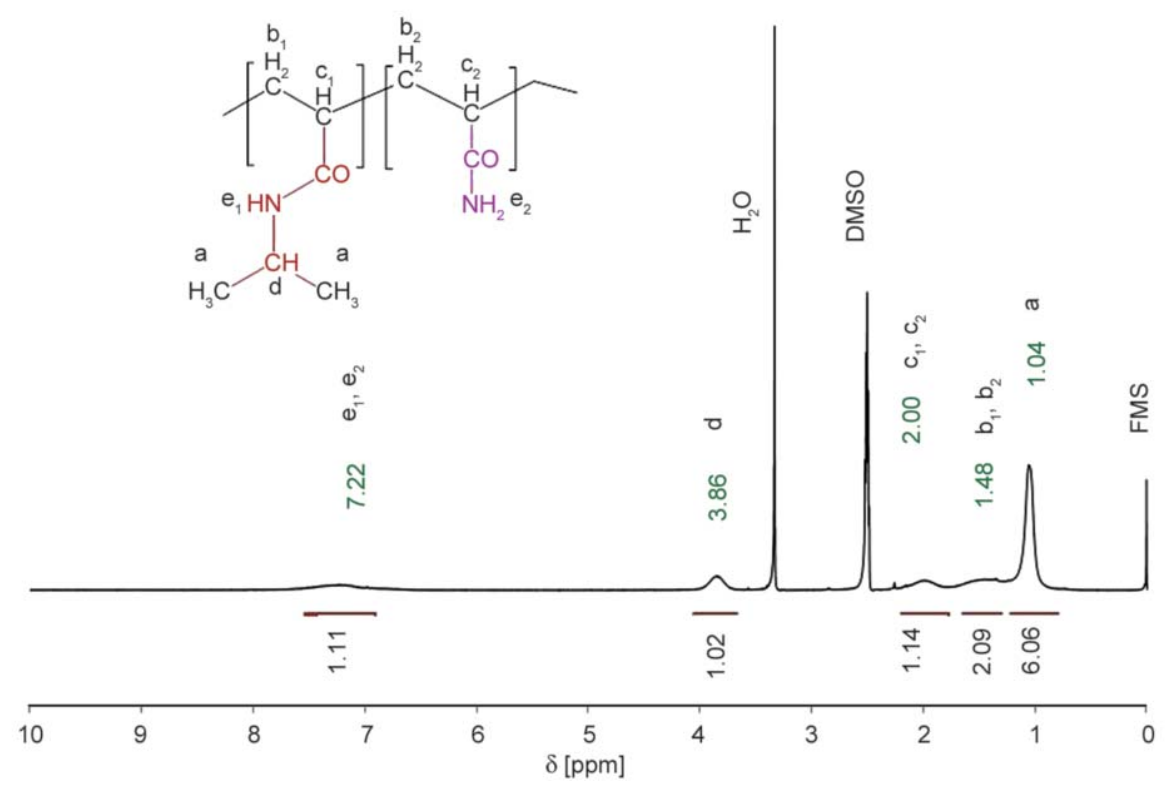

Figure 2. ${ }^{1} \mathrm{H}$ NMR spectrum of copolymer 7 in DMSO-d 6

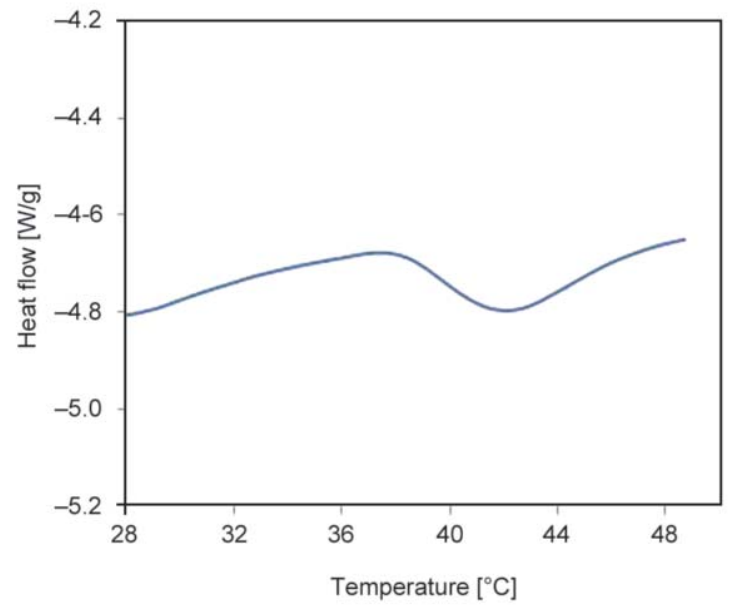

Figure 3. DSC curve of copolymer 7

culated by NMR for copolymer 7 was $89.5 \%$ NIPAM and $10.5 \% \mathrm{AM}$ which is well in agreement with the used feed composition (refer to Table 1). The DSC heating cycle curve of copolymer 7 in water solution $(50 \mathrm{mg} / \mathrm{mL})$ is shown in Figure 3. The minimum point indicates that the LCST of the copolymer at $41.2^{\circ} \mathrm{C}$ is very near to the cloud point observed (refer to Table 2).

\subsection{Grafting of copolymer on SBA-15 particles}

The SEM images of SBA-15 type mesoporous silica particles used in this work (Figure 5) show aggregates of several micrometers, consisting of primary microparticles of around 0.8 to $1 \mu \mathrm{m}$. The synthesis protocol followed for the grafting of random copolymer on mesoporous silica particles is shown in Fig- ure $4 \mathrm{a}$. In the first step of the reaction the reactivity of surface silanols on SBA-15 particles were exploited as anchoring points because they easily react with alkoxysilane groups of MPS.This step resulted in grafting of MPS on bare SBA-15 particles with polymerizable acryloxy groups [22]. In the second step in situ copolymerization of acryloxy-grafted particles with NIPAM and AM was carried out. In general silanol groups do not interfere with the chain propagation during the polymer formation. In fact, their reactivity is considerable in aqueous solvents (where they dissociate, with an isoelectric point around 23 ), but is much less in anhydrous organic solvents [23]. Structure of the polymer and its grafting by using the reactivity of surface silanols inside the pores are shown in Figure 4b. The coil-to-globule transition of the copolymer upon heating above LCST inside the pores is shown in Figure 4c.

\subsection{Characterization of poly(NIPAM-co-AM)/SBA particles}

The ATR-FTIR spectra of the starting bare SBA-15 porous silica particles, SBA-MPS particles and copolymer-grafted particles are shown in Figure 6. The spectrum of SBA-15 particles showed an intense broad band around $990-1000 \mathrm{~cm}^{-1}$ due to the stretching vibrations of $\mathrm{Si}-\mathrm{O}-\mathrm{Si}$ groups [24-26]. The spectra of MPS functionalized particles (inset zoomed area) showed two peaks at 1704 and $1630 \mathrm{~cm}^{-1}$ due to carbonyl $(\mathrm{C}=\mathrm{O})$ and $\mathrm{C}=\mathrm{C}$ stretching vibrations, respectively [27]. The copolymer-grafted particles (lower spectrum in inset zoomed area) showed peaks 

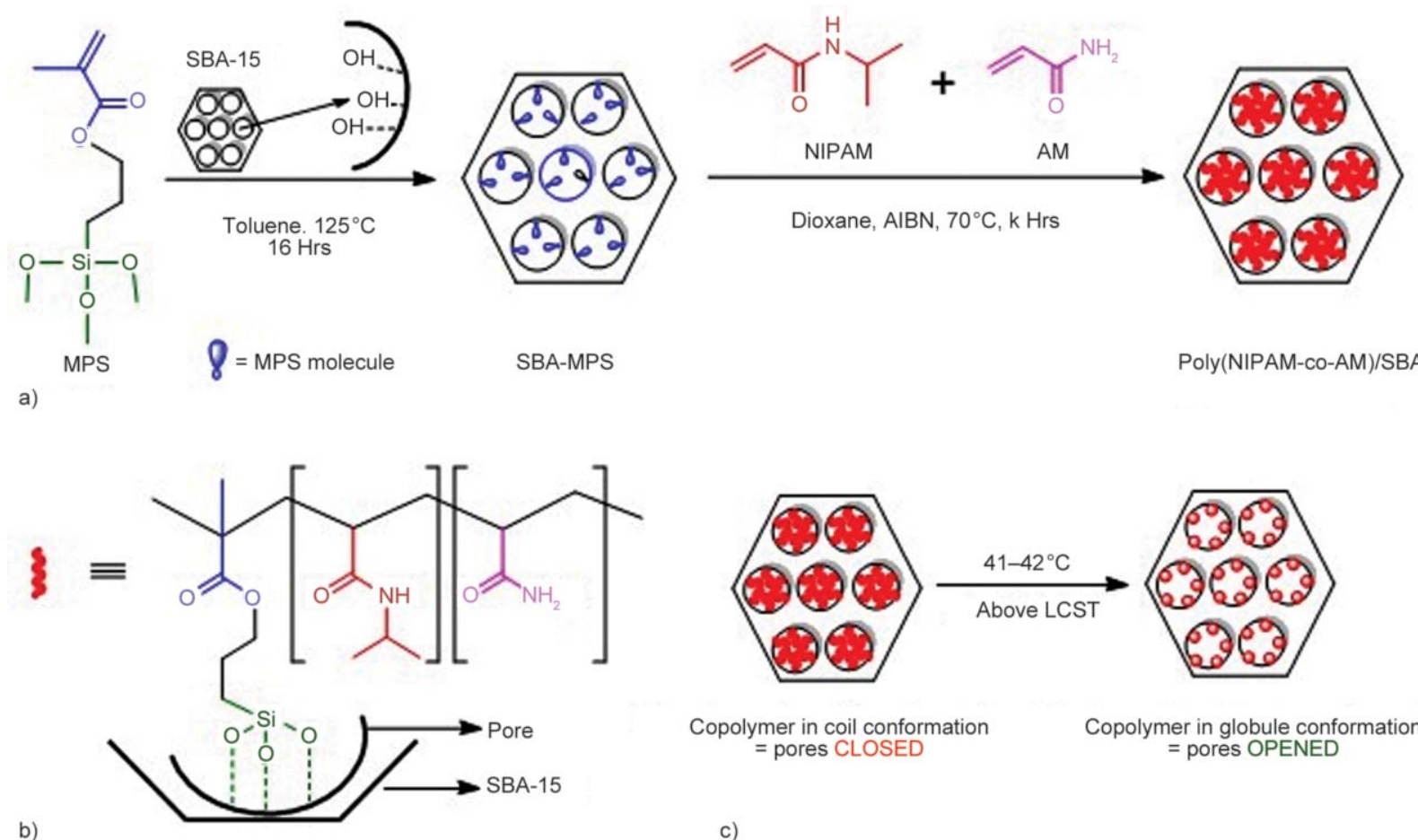

Poly(NIPAM-co-AM)/SBA

b)

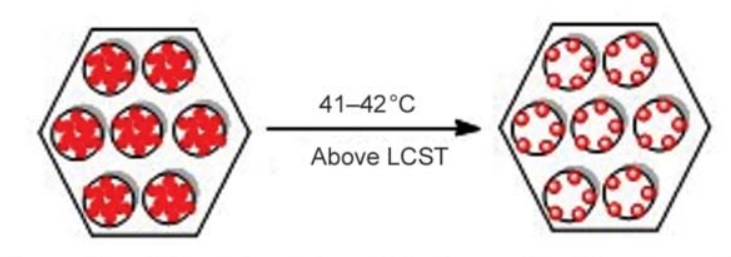

Copolymer in coil conformation $=$ pores $\mathrm{CLOSED}$

Copolymer in globule conformation $=$ pores OPENED

c)

Figure 4. a) Two-step grafting of poly(NIPAM-co-AM) on SBA particles, b) structure of the random copolymer grafted inside SBA pores and c) coil-to-globule transition of the copolymer inside pores

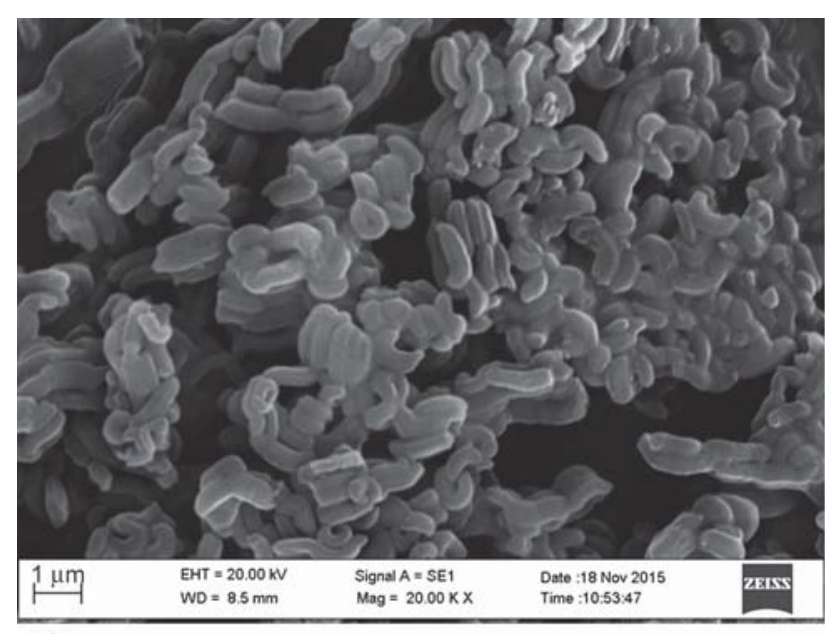

a)

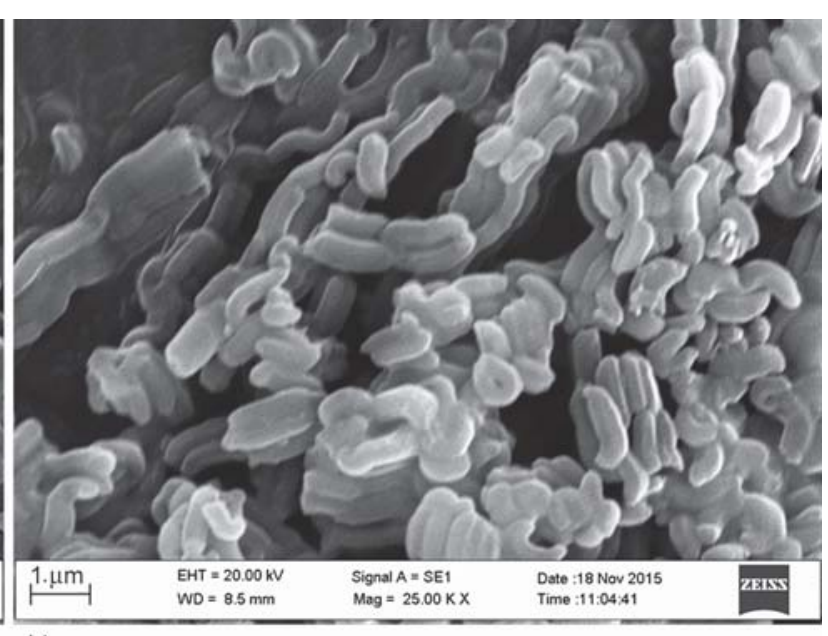

b)

Figure 5. SEM images ( $a$ and $b$ ) of SBA-15 particles used

at $1702,1668,1551,1460 \mathrm{~cm}^{-1}$ due to MPS carbonyl, secondary amide carbonyl, secondary amide $-\mathrm{N}-\mathrm{H}$ bending and $\mathrm{C}-\mathrm{H}$ asymmetric bending of the isopropyl group respectively [28, 29].

TGA curves of the SBA-15, SBA-MPS and poly(NIPAM-co-AM)/SBA samples are shown in Figure 7. SBA-15 particles show good thermal stability and upon programmed heating up to $800^{\circ} \mathrm{C}$ only $0.25 \%$ weight loss for the particles was observed which is due to the adsorbed humidity. The weight loss for SBA-MPS particles was $10.9 \%$ and for the poly(NIPAM-co-AM)/SBA sample was $14 \%$. The copolymer grafting percentage calculated was
$3.1 \%$. Results of the ATR-FTIR and thermal analysis confirmed grafting of the copolymer on the particles. The copolymer-grafted SBA particles were further characterized by nitrogen adsorption-desorption analysis. The adsorption-desorption isotherms obtained are shown in Figures 8a and 8b. The specific surface area for the SBA-15 particles calculated by BET analysis was $816 \mathrm{~m}^{2} / \mathrm{g}$, which was reduced to $622 \mathrm{~m}^{2} / \mathrm{g}$ for the copolymer-grafted particles. Due the grafting of the copolymer inside the mesopores, the pore volume was decreased from 1.14 to $0.96 \mathrm{~cm}^{3} / \mathrm{g}$ and the pore diameter (shown in Figure 9) was decreased from 56.3 to $54.9 \AA$ [30]. These results al- 


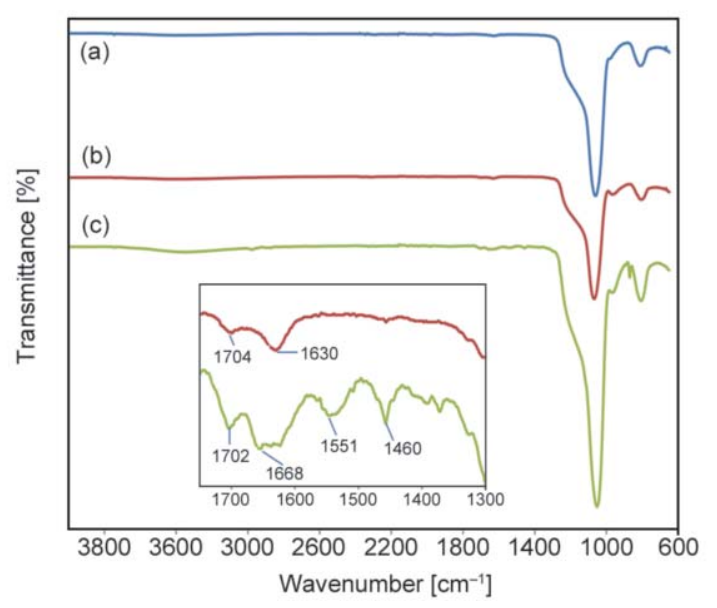

Figure 6. ATR-FTIR spectra of (a) SBA-15, (b) SBA-MPS and (c) poly(NIPAM-co-AM)/SBA particles

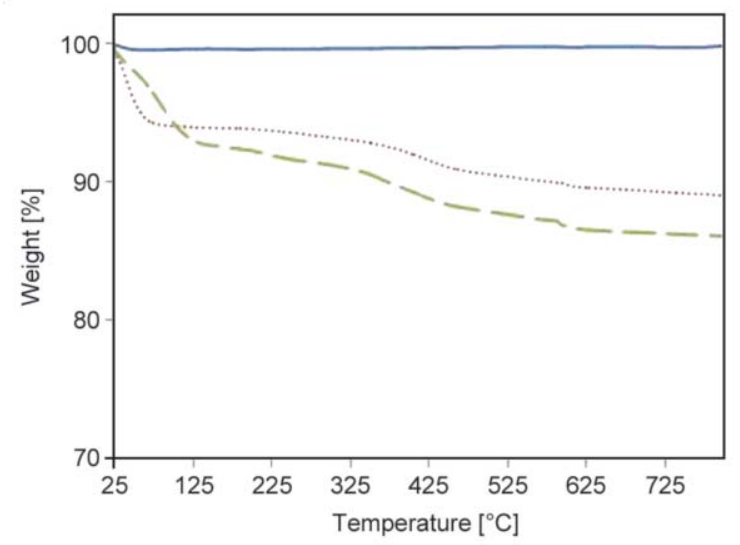

Figure 7. TGA curves of SBA-15 (solid line), SBA-MPS (dotted line) and poly(NIPAM-co-AM)/SBA (dashed line) particles
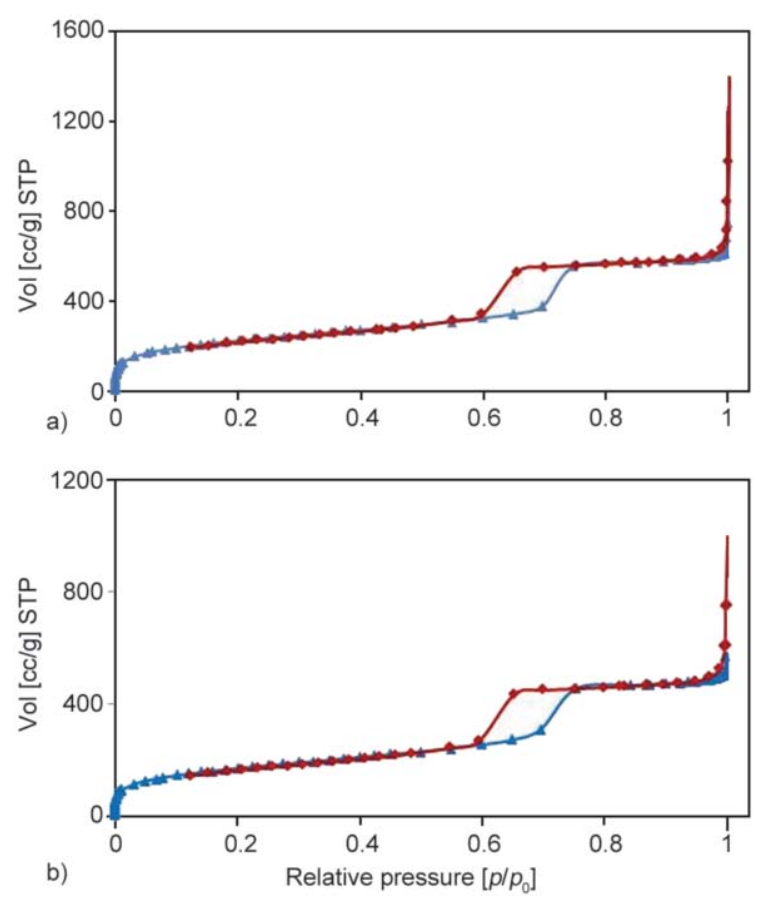

Figure 8. $\mathrm{N}_{2}$ adsorption (triangles) and desorption (squares) isotherms of a) SBA-15 and b) poly(NIPAM-coAM)/SBA particles

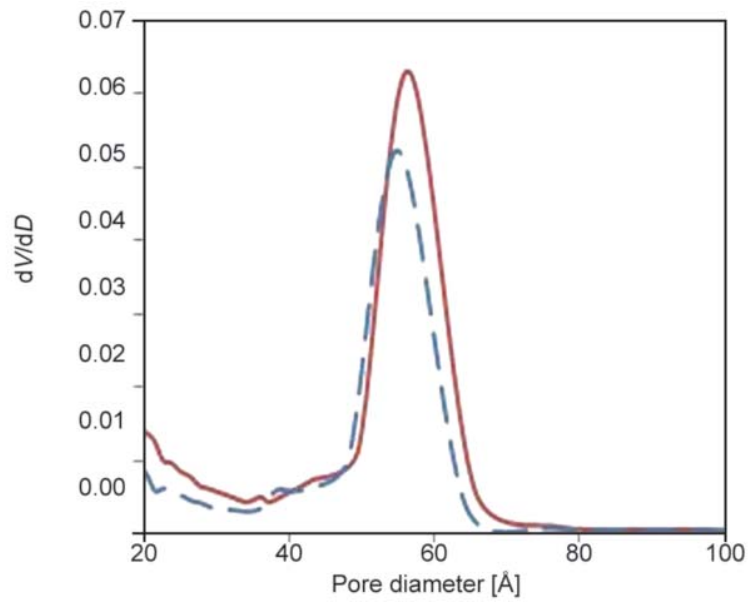

Figure 9. Pore size distribution curves of SBA-15 (solid line) and poly(NIPAM-co-AM)/SBA particles (dashed line)

together confirmed the effective grafting of the thermoresponsive copolymer inside the pores of the SBA-15 particles.

\subsection{Rutin loading and release tests}

Rutin (quercetin-3-O-rutinoside) was chosen as the model molecule for loading inside the porous particles. The SBA-15 type mesoporous silica particles were purposely chosen to prepare the delivery vehicle as they have large pores, hence big molecules such as rutin can easily enter and be loaded within the pores [31]. The obtained rutin loading was 35.3 and $27.9 \%$ for bare SBA-15 and poly(NIPAM-co-AM)/ SBA particles respectively (refer to Table 3). Rutin, being an unstable antioxidant is vulnerable to oxidation and undergoes degradation to form various products [32] hence its stability in the release medium was tested by monitoring its concentration by UVvisible spectroscopy (Figure 10). It was observed that rutin remains stable in the release medium over a

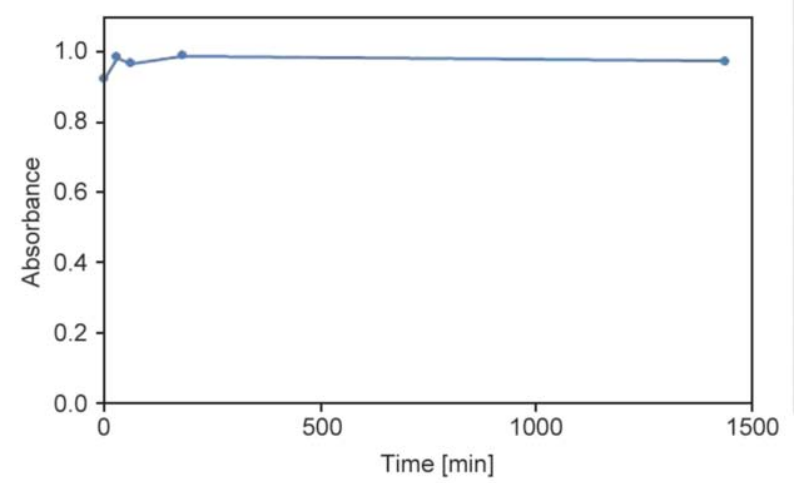

Figure 10. Stability of rutin in release medium phosphate buffer (pH 6.5)/DMSO (75/25 v/v) at $45^{\circ} \mathrm{C}$ 

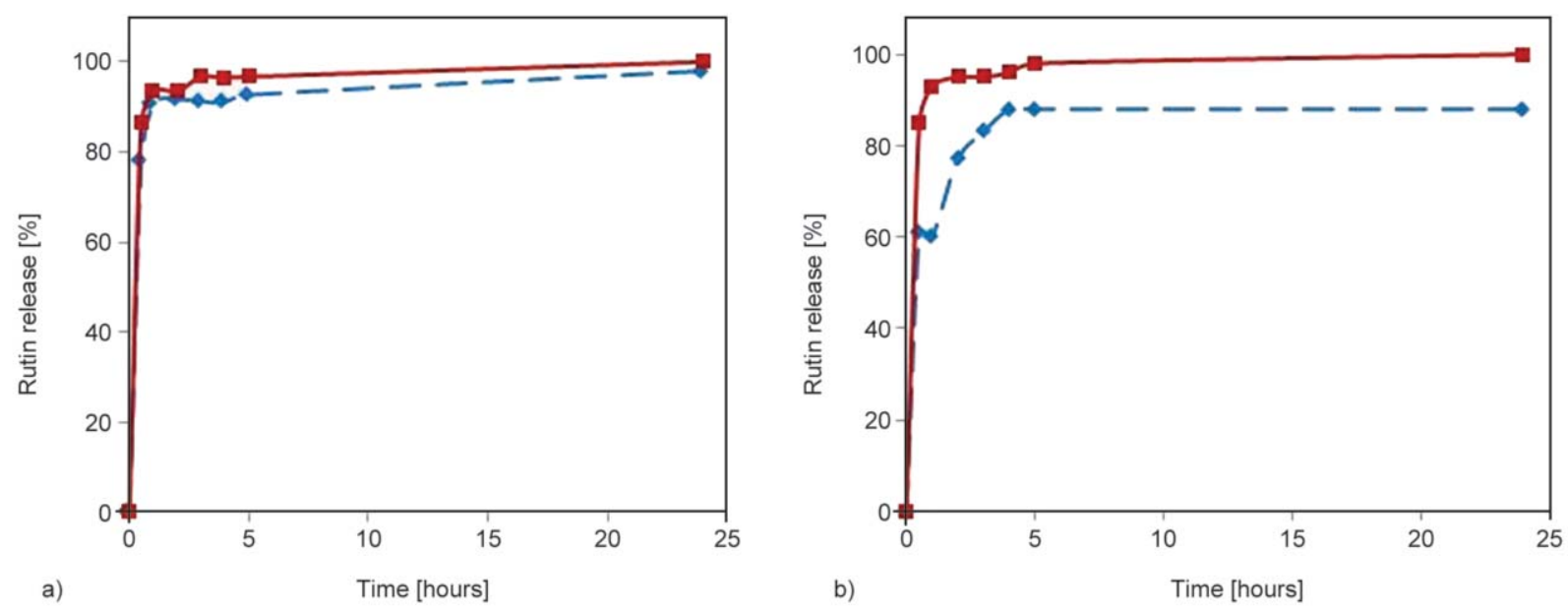

Figure 11. Rutin release curves from a) bare SBA-15 and b) poly(NIPAM-co-AM)/SBA particles, at $20^{\circ} \mathrm{C}$ (dotted line with diamonds) and $45^{\circ} \mathrm{C}$ (solid line with squares), respectively

period of 24 hours. This stability study confirms that the quantitative release of rutin calculated at each time interval (Figure 10) can be directly related to the concentration of rutin in the release medium. In the case of rutin-loaded SBA-15 (Figure 11a), a burst release was observed both at 20 and $45^{\circ} \mathrm{C}$. This can be related to the competition of the release medium (buffer solution) with the silica surface, displacing physically adsorbed rutin molecules [33, 34]. In the case of thermoresponsive copolymer-grafted SBA-15 particles a considerable difference of the quantitative release of rutin at two temperatures, below and above the LCST of the polymer, was observed (Figure 11b). Below the LCST the soluble extended copolymer chains block the immediate release of the rutin molecules by plugging the SBA pores and when the temperature is raised to $45^{\circ} \mathrm{C}$, which is well above the LCST of the copolymer, the polymer chains collapse to open the pores thus allowing the release of rutin. The initial burst release observed for bare SBA-15 particles was significantly reduced by the copolymer-grafted particles. The transition of the thermoresponsive copolymer from its hydrophilic coil conformation to hydrophobic globules applies a slightly hydrophobic character to the surface of the particles inside the mesopores which helps to minimise the interactions of the loaded molecules with the silica surface (silanols) and allows complete release of rutin. Additionally the hydrophobic copolymer layer also helps to minimize the readsorption of the released molecules back on the silica surface. Thus, release tests confirmed improved control of the hybrid thermoresponsive copolymergrafted particles over rutin release.

\section{Conclusions}

A series of thermoresponsive random copolymers of NIPAM with acrylamide were synthesized to tune the LCST of the copolymers. In situ copolymer grafting on mesoporous silica particles was carried out by replicating the optimized synthesis conditions for the selected copolymer with highest LCST. Effective grafting of the thermoresponsive copolymer on SBA-15 type porous silica particles was carried out to transform the particles into thermoresponsive dermal delivery systems. In vitro release tests performed by using rutin as the model molecule showed that the hybrid i.e. thermoresponsive copolymer-grafted porous silica particles are characterized by an improved control over rutin release. Burst release of the drug and readsoption of the drug was minimized. The synthesis of thermoresponsive copolymer-grafted porous material presented in this work proves to be an important step towards the transformation of porous materials into temperature-triggered drug delivery systems. Besides the results presented, the general importance of the work lies in the fact that the synthetic strategies reported can be applied easily to various other porous materials to transform them into temperature-triggered delivery systems.

\section{Acknowledgements}

Dr. Sushilkumar A. Jadhav thank MIUR, Italy, for financial support. The authors thank Dr. P. Quagliotto for NMR analyses. 


\section{References}

[1] Gandhi A., Paul A., Sen S. O., Sen K. K.: Studies on thermoresponsive polymers: Phase behaviour, drug delivery and biomedical applications. Asian Journal of Pharmaceutical Science, 10, 99-107 (2015).

https://doi.org/10.1016/j.ajps.2014.08.010

[2] Guan Y., Zhang Y.: PNIPAM microgels for biomedical applications: From dispersed particles to 3D assemblies. Soft Matter, 7, 6375-6384 (2011).

https://doi.org/10.1039/c0sm01541e

[3] Tan L., Liu J., Zhou W., Wei J., Peng Z. A.: A novel thermal and $\mathrm{pH}$ responsive drug delivery system based on ZnO@PNIPAM hybrid nanoparticles. Materials Science and Engineering: C, 45, 524-529 (2014).

https://doi.org/10.1016/j.msec.2014.09.031

[4] Yadavalli T., Ramaswamy S., Chandrashekharan G., Michael I., Therese H. A., Chennakesavulu R.: Dual responsive PNIPAM-chitosan targeted magnetic nanopolymers for targeted drug delivery. Journal of magnetism and Magnetic Materials, 380, 315-320 (2015). https://doi.org/10.1016/j.jmmm.2014.09.035

[5] Ward M. A., Georgiou T. K.: Thermoresponsive polymers for biomedical applications. Polymers, 3, 1215 1242 (2011). https://doi.org/10.3390/polym3031215

[6] Heskins M., Guillet J. E.: Solution properties of poly $(N$ isopropylacrylamide). Journal of Macromolecular Science: Part A Chemistry, 2, 1441-1455 (1968). https://doi.org/10.1080/10601326808051910

[7] Schild H. G.: Poly( $N$-isopropylacrylamide): Experiment, theory and application. Progress in Polymer Science, 17, 163-249 (1992).

https://doi.org/10.1016/0079-6700(92)90023-R

[8] Jain K., Vedarajan R., Watanabe M., Ishikiriyama M., Matsumi N.: Tunable LCST behavior of poly $(N$-isopropylacrylamide/ionic liquid) copolymers. Polymer Chemistry, 6, 6819-6825 (2015).

https://doi.org/10.1039/C5PY00998G

[9] Lee S-C., Chang J-Y.: Thermosensitive block copolymers consisting of poly( $N$-isopropylacrylamide) and star shape oligo(ethylene oxide). Bulletin of the Korean Chemical Society, 30, 1521-1525 (2009).

https://doi.org/10.5012/bkcs.2009.30.7.1521

[10] Kwon S., Singh R. K., Perez R. A., Abou Neel E. A., Kim H W., Chrzanowski W.: Silica-based mesoporous nanoparticles for controlled drug delivery. Journal of Tissue Engineering, 4, 2041731413503357/12041731413503357/18 (2013).

https://doi.org/10.1177/2041731413503357

[11] Ugazio E., Gastaldi L., Brunella V., Scalarone D., Jadhav S. A., Oliaro-Bosso S., Zonari D., Berlier G., Miletto I., Sapino S.: Thermoresponsive mesoporous silica nanoparticles as a carrier for skin delivery of quercetin. International journal of Pharmaceutics, 511, 446-454 (2016).

https://doi.org/10.1016/j.ijpharm.2016.07.024
[12] Brunella V., Jadhav S. A., Miletto I., Berlier G., Ugazio E., Sapino S., Scalarone D.: Hybrid drug carriers with temperature-controlled on-off release: A simple and reliable synthesis of PNIPAM-functionalized mesoporous silica nanoparticles. Reactive and Functional Polymers, 98, 31-37 (2016).

https://doi.org/10.1016/j.reactfunctpolym.2015.11.006

[13] Baek S. M., Singh R. K., Kim T-H., Seo J-W., Shin U. S., Chrzanowski W., Kim H-W.: Triple hit with drug carriers: $\mathrm{pH}$ - and temperature-responsive theranostics for multimodal chemo- and photothermal therapy and diagnostic applications. ACS Applied Materials and Interfaces, 8, 8967-8979 (2016).

https://doi.org/10.1021/acsami.6b00963

[14] Hong C-Y., Li X., Pan C-Y.: Smart core-shell nanostructure with a mesoporous core and a stimuli-responsive nanoshell synthesized via surface reversible addition-fragmentation chain transfer polymerization. Journal of Physical Chemistry C, 112, 15320-15324 (2008). https://doi.org/10.1021/jp805028z

[15] Hosseinzadeh H., Nassiri-Asl M. J.: Review of the protective effects of rutin on the metabolic function as an important dietary flavonoid. Journal of Endocrinological Investigation, 37, 783-788 (2014).

https://doi.org/10.1007/s40618-014-0096-3

[16] Chua L. S.: A review on plant-based rutin extraction methods and its pharmacological activities. Journal of Ethnopharmacology, 150, 805-817 (2013).

https://doi.org/10.1016/j.jep.2013.10.036

[17] Eeckman F., Moës A. J., Amighi K.: Synthesis and characterization of thermosensitive copolymers for oral controlled drug delivery. European Polymer Journal, 40, 873-881 (2004).

https://doi.org/10.1016/j.eurpolymj.2003.11.010

[18] Furyk S., Zhang Y., Ortiz-Acosta D., Cremer P. S., Bergbreiter D. E.: Effects of end group polarity and molecular weight on the lower critical solution temperature of poly( $N$-isopropylacrylamide). Journal of Polymer Science Part A: Polymer Chemistry, 44, 1492-1501 (2006). https://doi.org/10.1002/pola.21256

[19] Hao L., Yang H., Lei Z.: Synthesis and properties of thermo-responsive macroporous PAM-co-PNIPAM microspheres. Material Letters, 70, 83-85 (2012).

https://doi.org/10.1016/j.matlet.2011.11.112

[20] Schönhoff M., Larsson A. Welzel P. B., Kuckling D.: Thermoreversible polymers adsorbed to colloidal silica: A ${ }^{1} \mathrm{H}$ NMR and DSC study of the phase transition in confined geometry. Journal of Physical Chemistry B, 106, 7800-7808 (2002).

https://doi.org/10.1021/jp0155381

[21] Sun S., Wu P., Zhang W., Zhang W., Zhu X.: Effect of structural constraint on dynamic self-assembly behavior of PNIPAM-based nonlinear multihydrophilic block copolymers. Soft Matter, 9, 1807-1816 (2013).

https://doi.org/10.1039/C2SM27183D 
[22] Jadhav S. A., Brunella V., Scalarone D.: Polymerizable ligands as stabilizers for nanoparticles. Particle and Particle Systems Characterization, 32, 417-428 (2015). https://doi.org/10.1002/ppsc.201400074

[23] Jadhav S. A., Miletto I., Brunella V., Berlier G., Scalarone D.: Controlled post-synthesis grafting of thermoresponsive poly( $\mathrm{N}$-isopropylacrylamide) on mesoporous silica nanoparticles. Polymers for Advanced Technologies, 26, 1070-1075 (2015). https://doi.org/10.1002/pat.3534

[24] Li L-L., Sun H., Fang C-J., Xu J., Jin J-Y., Yan C-H.: Optical sensors based on functionalized mesoporous silica SBA-15 for the detection of multianalytes $\left(\mathrm{H}^{+}\right.$ and $\mathrm{Cu}^{2+}$ ) in water. Journal of Materials Chemistry, 17, 4492-4498 (2007). https://doi.org/10.1039/b708857d

[25] Zhang D., Li J.: Ordered SBA-15 mesoporous silica with high amino-functionalization for adsorption of heavy metal ions. Chinese Science Bulletin, 58, 879 883 (2013).

https://doi.org/10.1007/s11434-012-5594-0

[26] Borodko Y., Ager J. W., Marti G. E., Song H., Niesz K., Somorjai G. A.: Structure sensitivity of vibrational spectra of mesoporous silica SBA-15 and Pt/SBA-15. Journal of Physical Chemistry B, 109, 17386-17390 (2005). https://doi.org/10.1021/jp051801x

[27] Chinthamanipeta P. S., Kobukata S., Nakata H., Shipp D. A.: Synthesis of poly(methyl methacrylate)-silica nanocomposites using methacrylate-functionalized silica nanoparticles and RAFT polymerization. Polymer, 49, 5636-5642 (2008).

https://doi.org/10.1016/j.polymer.2008.10.018

[28] Chen J., Liu M., Chen C., Gong H., Gao C.: Synthesis and characterization of silica nanoparticles with welldefined thermoresponsive PNIPAM via a combination of RAFT and click chemistry. ACS Applied Materials and Interfaces, 3, 3215-3223 (2011).

https://doi.org/10.1021/am2007189
[29] Yang Y., Yan X., Cui Y., He Q., Li D., Wang A., Fei J., Li J.: Preparation of polymer-coated mesoporous silica nanoparticles used for cellular imaging by a 'graft-from' method. Journal of Materials Chemistry, 18, 5731-5737 (2008).

https://doi.org/10.1039/b811573g

[30] Reichhardt N. V., Giullet-Nicolas R., Thommes M., Klösgen B., Nylander T., Kleitez F., Alfredsson V.: Mapping the location of grafted PNIPAAM in mesoporous SBA-15 silica using gas adsorption analysis. Physical Chemistry Chemical Physics, 14, 5651-5661 (2012). https://doi.org/10.1039/c2cp22523a

[31] Ritter H., Ramm J. H., Brühwiler D.: Influence of the structural properties of mesoporous silica on the adsorption of guest molecules. Materials, 3, 4500-4509 (2010).

https://doi.org/10.3390/ma3084500

[32] Friedman M., Jürgens H. S.: Effect of pH on the stability of plant phenolic compounds. Journal of Agricultural and Food Chemistry, 48, 2101-2110 (2000). https://doi.org/10.1021/jf990489j

[33] Li J., Wang H., Li H., Xu L., Guo Y., Lu F., Pan W., Li S.: Mutual interaction between guest drug molecules and host nanoporous silica xerogel studied using central composite design. International Journal of Pharmaceutics, 498, 32-39 (2016). https://doi.org/10.1016/j.ijpharm.2015.12.005

[34] Malfanti A., Miletto I., Bottinelli E., Zonari D., Blandino G., Berlier G., Arpicco S.: Delivery of gemcitabine prodrugs employing mesoporous silica nanoparticles. Molecules, 21, 522/1-522/15 (2016). https://doi.org/10.3390/molecules21040522 